\section{Rede Nacional de Bancos de Leite Humano: gênese e evolução}

\section{Human Milk Banks National Network: genesis and evolution}

Paulo Ricardo da Silva Maia 1 João Aprígio Guerra de Almeida 2 Franz Reis Novak 3 Danielle Aparecida da Silva 4

\footnotetext{
1-4 Banco de Leite Humano. Instituto Fernandes Figueira. Fundação Oswaldo Cruz. Av. Rui Barbosa, 716, 1. andar. Flamengo. Rio de Janeiro, RJ, Brasil. CEP: 22.250-020. E-mail: pmaia@fiocruz.br
}

\begin{abstract}
Objectives: establishment and progress analysis of the Human Milk Banks National Network in Brazil. Determinant elements are highlighted for their acknowledged participation and influence on the state policy for the sector.

Methods: content analysis of primary documental sources generated by official organizations and institutions offering Human Milk Banks services were analyzed, as well as thesis, dissertations, books and scientific articles focusing on the subject. Social perceptions and concerns were assessed and the conclusion was that with time they suffered changes and depending on the historical moment considered, actors and social groups have conveyed different meanings to the service.

Results: knowledge appears to give conductivity to the network. That is, analysis performed of the Banks creation and progress, identified that in addition to the social actors involved, there are knowledge production dynamics that should be understood.

Conclusions: it's possible to identify a potential Network contribution to promote social change and to formulate public policies aiming at women and children's health.
\end{abstract}

Key words Milk banks, History, Knowledge

\section{Resumo}

Objetivos: analisar a gênese e evolução da Rede Nacional de Bancos de Leite Humano no Brasil. São destacados os elementos determinantes de sua reconhecida participação e influência na formulação da política estatal para o setor.

Métodos: foram realizadas análises de conteúdo de fontes documentais primárias geradas pelos órgãos oficiais e instituições mantenedoras de Bancos de Leite Humano, de teses, dissertações, livros e artigos científicos, que versam sobre a temática. Identificou-se que as percepções e construções sociais acerca destas unidades de serviço sofreram flutuações ao longo do tempo, e a depender do momento histórico que se considere, atores e grupos sociais lhes atribuíram diferentes significados.

Resultados: o conhecimento aparece como elemento que confere conectividade à Rede. Ou seja, a análise realizada, da gênese e evolução dos Bancos, permite identificar que além dos movimentos dos atores sociais envolvidos, há uma dinâmica da produção do conhecimento que deve ser entendida.

Conclusões: é possível identificar a potencial contribuição da Rede para promoção de transformações sociais e para a formulação de políticas públicas voltadas para área da saúde da mulher e da criança.

Palavras-chave Bancos de leite, História, Conhecimento 


\section{Introdução}

A política pública de saúde, voltada para o incentivo à amamentação tem, ao longo das últimas décadas, fortalecido a importância dos bancos de leite humano (BLH). Essas unidades configuram-se como locais privilegiados para as ações de incentivo ao aleitamento materno no território nacional. Contudo, vale destacar que as percepções e construções sociais acerca de tais unidades de serviço sofreram flutuações ao longo do tempo, e a depender do momento histórico que se considere, atores e grupos sociais lhes atribuíram diferentes significados. Assim foram caracterizadas tanto como estruturas de apoio às situações de excepcionalidade do desmame comerciogênico, como unidades de atendimento a serviço da amamentação.1,2

O primeiro BLH do Brasil foi implantado em outubro de 1943 no então Instituto Nacional de Puericultura, atualmente Instituto Fernandes Figueira (IFF). O seu principal objetivo era coletar e distribuir leite humano visando atender os casos considerados especiais, a exemplo da prematuridade, perturbações nutricionais e alergias a proteínas heterólogas. Com essa mesma perspectiva, entre a década de quarenta e o início dos anos oitenta do século passado, foram implantadas mais cinco unidades no país. Contudo, foi com o desenvolvimento do Programa Nacional de Incentivo ao Aleitamento Materno, sobretudo a partir de 1985 , que os BLH passaram a assumir um novo papel no cenário da saúde pública brasileira, transformandose em elementos estratégicos para as ações de promoção, proteção e apoio à amamentação.1,2

Esse novo modelo induziu um período de franca expansão e viria mais tarde a se transformar na maior rede mundial de bancos de leite humano. No momento atual, dela fazem parte mais de 180 unidades operando em todo território nacional. O Ministério da Saúde tem projeto para implantação de dez novas unidades no curto prazo.

Para compreender a gênese e a evolução da Rede Nacional de Bancos de Leite Humano (REDEBLH) é necessário resgatar a historicidade desses serviços de saúde, ao longo de seus sessenta anos de existência no Brasil. Em especial, no que concerne aos elementos determinantes de sua reconhecida participação e influência tanto na formulação da política estatal para o setor como na geração e disseminação do conhecimento científico produzido na área.

\section{Métodos}

Utilizou-se o referencial teórico-metodológico da pesquisa qualitativa em saúde. ${ }^{3}$ Parte-se portanto da premissa epistêmica de que o conhecimento é produzido numa interação dinâmica entre o sujeito e o objeto do conhecimento. Com o intuito de buscar o significado das ações sociais dos atores envolvidos na construção da REDEBLH foi realizada pesquisa bibliográfica. Foram também analisadas teses e dissertações defendidas na pós-graduação do Instituto Fernandes Figueira da Fundação Oswaldo Cruz (FIOCRUZ). Para seleção dos trabalhos analisados adotou-se como critério os estudos cujo objeto de investigação estava centrado nas temáticas de bancos de leite humano ou aleitamento materno no período de 1998 a 2004. Utilizou-se o catálogo de teses da FIOCRUZ para localização dos documentos.

A análise revelou que a trajetória dos BLH no Brasil pode ser dividida em três períodos distintos, assim demarcada: 1943/1984 - fase inicial de consolidação com a implantação da primeira unidade; 1985/1997 - ampliação da forma de atuação, com a incorporação de atividades de promoção, proteção e apoio à amamentação; e a partir de 1998 - desenvolvimento do projeto da Rede Nacional cujo modelo instala um processo de crescimento pautado na descentralização e na construção de competência técnica nos estados e municípios. Um panorama desses diferentes momentos passa a ser descrito a seguir.

\section{Resultados e Discussão}

Os precursores na implantação de BLH do país em 1943 foram os professores Mário Olinto e Adamastor Barbosa do Departamento Nacional da Criança. Contudo, registros revelam que a primeira iniciativa de manipulação de leite humano ordenhado no Brasil teve lugar no Lactário de Leite Humano, construído por Martagão Gesteira, no Abrigo Maternal da cidade de Salvador, na Bahia. ${ }^{1}$

Os BLH foram originalmente projetados para atender casos especiais, em que o leite humano era considerado imprescindível, muito mais por suas propriedades farmacológicas do que por suas qualidades nutricionais. Assim o leite humano destinavase tão somente às situações de emergência que não podiam ser solucionadas com a alimentação artificial, que era colocada como primeira alternativa. 4

Não havia competição entre o leite humano distribuído e os produtos industrializados. Do ponto 
de vista epidemiológico, tinha-se que $85 \%$ dos óbitos, decorrentes de desnutrição nos lactentes desmamados, estavam relacionados ao uso de alimentação artificial. Dessa forma, era necessário um "estoque" de leite humano que pudesse ser disponibilizado para atender aquela demanda. Essa questão, associada à questionável resolutividade das opções alimentares alternativas, justificava a necessidade de um BLH.1,2,4

Em decorrência, os BLH teriam surgido como uma alternativa capaz de preencher a lacuna deixada pela incapacidade de resposta dos produtos destinados à alimentação do lactente, de forma discreta e bem delimitada, sem nenhuma perspectiva de construir avanços nesse campo para além do que as fórmulas fossem capazes de possibilitar. ${ }^{4}$

O principal objetivo dos BLH, por mais de quarenta anos (de 1943 a 1985), foi a coleta. A doação não resultava de um processo voluntário e consciente. Ao contrário, havia casos em que a doadora era remunerada de acordo com a quantidade de leite disponibilizado, operando numa lógica com evidências comerciais. Eram também adotados rigorosos critérios para a seleção das doadoras. Além do exame físico geral e inspeção minuciosa com ênfase para doenças contagiosas, efetuava-se o exame ginecológico na busca de outras enfermidades. $1,2,4$

Em relação aos cuidados dispensados ao leite e à sua manipulação, era recomendado rigor asséptico em todas as etapas, desde a ordenha até o consumo.

O leite era distribuído preferencialmente na forma de produto cru, sem receber qualquer tipo de tratamento. Entretanto, em decorrência do grande volume de leite coletado, fez-se necessário introduzir o tratamento térmico, que era conduzido em equipamento de esterilização de mamadeiras, em banho-maria por 20 minutos. 5

A concepção de funcionamento tinha idealmente a intenção de ser um órgão de proteção social, com objetivo de preservar e garantir os interesses da doadora e de seu filho. Não havia expectativa de lucro, mas estimulava a prática da amamentação natural por meio de recompensa oferecida à nutriz pelo leite doado. Entretanto, é importante entender a distância existente entre a intenção expressa na definição do modelo e o que ele próprio possibilitou na prática. A desfavorável realidade socioeconômica das doadoras contribuía para a comercialização do leite que, para elas, se apresentava como forma de complementação de seu sustento e da família. Essa prática teria inclusive contribuído para estimular a gravidez. Na realidade, os BLH operavam basicamente nos processos de coleta e distribuição, rele- gando as ações de estímulo à amamentação a um plano secundário.

Ao longo dos anos 80 estrutura-se um novo modelo. Ocorre também importante expansão do número de BLH instalados no Brasil. Nesse crescimento desempenhou um importante papel o Programa Nacional de Incentivo ao Aleitamento Materno (PNIAM). Com a formalização do Grupo Técnico de Bancos de Leite Humano em 1984, teve início um processo de institucionalização de experiências até então isoladas. Três anos mais tarde seria elaborado o primeiro documento oficial de recomendações técnicas, que serviu de base para elaboração da primeira legislação federal, publicada na forma de portaria pelo Ministério da Saúde, demonstrando oficialmente a formalização de um processo de articulação das ações dos BLH com o aparelho de Estado. 6

Também nesse período, foi criado o Centro de Referência Nacional em Bancos de Leite Humano, um projeto de parceria entre a área da criança do Ministério da Saúde e a Fundação Oswaldo Cruz. Esse projeto viabilizou ações de desenvolvimento tecnológico, criando opções de baixo custo, centradas no processamento e no controle de qualidade do leite humano que foram gradualmente sendo incorporadas às rotinas.

Da mesma forma, estratégica foi a preparação e formação de quadros técnicos, em diversos graus de complexidade, para atuarem nos BLH do Brasil. Assim, havia o entendimento de que a radicalização na formação de quadros técnicos capazes era fundamental para reverter o quadro de desmame precoce. 1

De forma complementar, porém igualmente estratégica para o processo de estruturação dos BLH, foi organizado em 1992, o primeiro Encontro Nacional de Bancos de Leite Humano no Rio de Janeiro. Esse evento marcou a definição de um planejamento participativo e de um modelo genuíno de gestão, estabelecendo assim os alicerces da Rede Nacional de Bancos de Leite Humano.

Dando seguimento à lógica de planejamento participativo, foi organizado em 1995, o II Encontro Nacional de Bancos de Leite Humano. Naquele momento constatou-se haver um baixo nível de investimento público para fomento às ações dos BLH. Como alternativa de captação de recursos foi sugerida pela plenária a criação de uma organização não governamental. Tal iniciativa só não prosperou em função da retomada, por parte do Ministério da Saúde, de uma política considerada mais adequada às necessidades do setor. 1

Mais adiante, em julho de 1998, foi realizado em Brasília o I Congresso Brasileiro de Bancos de Leite 
Humano. Dessa forma, criava-se importante fórum para compartilhamento do conhecimento produzido. Esse evento produziu fatos que influenciariam, de forma contundente, o futuro da organização dos BLH no país, destacando-se entre eles: a consolidação da parceria com a Vigilância Sanitária Nacional e Estaduais; a discussão sobre o papel central da mulher no processo de amamentação e, a reafirmação da importância da qualificação dos profissionais. ${ }^{1}$

Ainda em 1998, foi criada pelo Ministério da Saúde, através do Centro de Referência Nacional da Fundação Oswaldo Cruz, a Rede Nacional de Bancos de Leite Humano. Tal fato significou, por um lado, importante decisão de política pública no campo da saúde e, de outro, sedimentou um novo modelo de gestão mais apropriado à realidade de expansão que se verificava naquele momento. A idéia de trabalho em rede se apresentava como solução apropriada. 7

Esta nova lógica operacional também contribuiu para a expansão das atividades da REDEBLH para além das fronteiras do território nacional.

A redução da mortalidade infantil foi seu objetivo estratégico e, portanto, a grande prioridade de sua atuação.

Para consecução dos objetivos da REDEBLH, o Centro de Referência Nacional, localizado no Rio de Janeiro, articula-se com cada Centro de Referência Estadual e suas respectivas comissões. As decisões são compartilhadas com as representações dos BLH localizadas em outros municípios.

$\mathrm{Na}$ articulação da REDEBLH, a informação e o conhecimento se tornam estratégicos. O Centro de Referência Nacional, sede da Rede, busca soluções para os problemas apontados em sua área de atuação. Dessa forma, as atividades acadêmicas desenvolvidas na sede da REDEBLH, buscam construir o conhecimento dito eficiente, capaz de promover as transformações sociais necessárias à melhoria da qualidade da saúde. 1

Em continuidade ao novo processo de estruturação das ações dos BLH, foi realizada em 1999 a primeira reunião nacional dos Centros de Referência Estaduais. A formulação de diretrizes para um novo programa de qualificação de recursos humanos, com base no curso de "Processamento e Controle de Qualidade de Leite Humano", foi considerada um dos importantes resultados do evento. 1

O desenvolvimento tecnológico também ocorreu na área da informação e comunicação. Em projeto de parceria com o Centro de Informação Científica e Tecnológica da Fundação Oswaldo Cruz (FIOCRUZ) foi criado, em 1998, o site da
REDEBLH - www.redeblh.fiocruz.br. A idéia era ampliar as oportunidades de acesso ao conhecimento e informação.

A projeção internacional do sucesso da experiência brasileira passou a ser decorrência natural, iniciando-se por países sul-americanos. Através de um programa de cooperação técnica, estabelecido entre o Ministério da Saúde, a FIOCRUZ e o Governo venezuelano, três BLH já foram implantados. Além disso, programas de cooperação estão sendo estabelecidos com a Universidade Central daquele país, para transferência de tecnologia.

No ano de 2003 teve início um processo estruturado de ampliação da REDEBLH. No momento, dando prioridade para a América Latina, estão em andamento mais duas iniciativas internacionais. Como decorrência, no Equador está sendo implantado o primeiro BLH do país, na Maternidade Isidro Ayora em Quito. No Uruguai também foram implantadas duas unidades.

A REDEBLH contribui para o compartilhamento do conhecimento em seu âmbito de atuação. Neste sentido, vale destacar a organização e realização do I Congresso Internacional de Bancos de Leite Humano e do II Congresso Brasileiro de Bancos de Leite Humano, ocorridos em 2000, na cidade de Natal, no Rio Grande do Norte. Na oportunidade, estiveram presentes profissionais vinculados a BLH de vários países. Esse evento caracterizou-se, no cenário internacional, como momento de afirmação da posição de vanguarda do Brasil na geração de conhecimento na área.

Todos estes aspectos conferem à REDEBLH, no que concerne ao seu modelo de atuação, uma posição diferenciada de outras experiências. Difere por ter uma atuação voltada para o incentivo à amamentação e também por trabalhar o leite humano na perspectiva dos referenciais da tecnologia de alimentos. ${ }^{1}$

Há consenso de que os resultados positivos alcançados pela REDEBLH foram decorrentes dos investimentos realizados no âmbito da pesquisa e do desenvolvimento tecnológico. Assim, esses referenciais foram o eixo temático do III Congresso Brasileiro de Bancos de Leite Humano, realizado em 2002 na cidade de Petrópolis, no Rio de Janeiro, que consolidou as bases de dois importantes programas da Rede Nacional - O Programa Nacional de Qualidade em Bancos de Leite Humano e o Sistema de Gestão Rede BLH - on line.

Em 2005, marcando os 20 anos de política pública em BLH, foi realizado em Brasília, no mês de maio, o II Congresso Internacional de Bancos de Leite Humano, concomitantemente ao IV Congresso Brasileiro. Além da oportunidade de mais uma vez 
viabilizar o compartilhamento do conhecimento, esse evento deu início a um processo de discussão com a finalidade de construção coletiva da Rede Latino-americana de BLH.

Em paralelo temporal ao processo de institucionalização da REDEBLH, via novo modelo de gestão, ocorria um movimento de consolidação das atividades de ensino e pesquisa na área da amamentação e bancos de leite, na busca da legitimação e sustentação acadêmica da REDEBLH. Tal fato foi bastante perceptível no espaço acadêmico circunscrito pela pós-graduação em Saúde da Mulher e da Criança do Instituto Fernandes Figueira, sede da Rede.

Levando-se em conta o eixo cronológico da produção acadêmica vinculada à linha de pesquisa em BLH nos cursos de mestrado e doutorado da referida pós-graduação, ou seja, do conhecimento científico produzido na sede da REDEBLH, é possível entender sua importância como elemento de legitimação. Para uma visão mais detalhada e, ao mesmo tempo panorâmica do conhecimento gerado, foram analisadas as contribuições das teses e dissertações defendidas nos últimos anos.

A tese de Almeida 8 tem a marca de uma das produções científicas de maior impacto na área de estudo nos tempos recentes. Num movimento inovador, o autor traz para a discussão o híbrido natureza-cultura como fundamento epistemológico para repensar questões sobre amamentação, configurando, dessa forma, um dos mais robustos referenciais teóricos que vem influenciando grande parte da produção literária e acadêmica nesse campo.

Segue nesta linha a tese de doutorado de Souza. ${ }^{9}$ Sustentada nos conceitos de transcendência e imanência, o autor desenvolveu uma pesquisa qualitativa, partindo de análise documental de fontes primárias. O estudo apresenta três principais eixos de discussão: o paradigma da amamentação desde o Brasil-colonial até os argumentos científicos atuais, a seguir analisa as construções acadêmicas no século XIX e século XX e, por fim, investiga a relação entre a propaganda e a construção do conhecimento e as estratégias de marketing utilizadas pela indústria de alimentos para lactentes. Sua conclusão é de que a pediatria brasileira, ao longo de sua trajetória, tem lidado preponderantemente com a amamentação como fenômeno natural, recortando-a enquanto objeto de estudo sob a égide das leis transcendentes. Ferreira 10 focaliza seu estudo partindo do pressuposto de que apenas o conhecimento biológico sobre amamentação é insuficiente para que a orientação e apoio às mulheres-mães, que buscam apoio, atinjam os resultados esperados. Seu objetivo é compreender o processo de decisão da mulher, mãe do bebê prematuro internado, em unidade de tratamento intensivo neonatal, sobre a alimentação de seu filho após a alta hospitalar. As conclusões apontam que essa decisão contém elementos dinâmicos e interdependentes, que culminam em duas categorias - tendo medo e temendo a perda de peso. O estudo indica, ainda, que a mulher se mostra sujeita a influências advindas de sua vivência anterior, de experiências familiares e de outras mulheres, bem como de orientações de profissionais.

De igual importância no que diz respeito à geração do conhecimento neste período, são os trabalhos relacionados ao assistir em amamentação e BLH. O estudo de Souza, 11 discute as representações sociais em aleitamento junto a profissionais de saúde e puérperas que participaram do programa Iniciativa Hospital Amigo da Criança (IHAC) comparando com grupos que dela não fizeram parte. Identifica como aspectos importantes as diferenças entre as representações sociais dos profissionais dos dois grupos e a similaridade das representações entre as mulheres estudadas. Tal investigação é importante, na medida em que oferece oportunidade de reflexão sobre o funcionamento e resultados de um programa oficial do Ministério da Saúde e ao mesmo tempo abre espaço para estudos de natureza qualitativa, utilizando as representações sociais como instrumento teórico-metodológico. Ramos, 12 também utilizando o referencial teórico-metodológico das representações sociais, investigou as alegações maternas para o desmame e a percepção sobre a assistência em amamentação, entre mulheres atendidas na Maternidade Dona Evangelina Rosa, em Teresina, Piauí, um Hospital Amigo da Criança. Com relação à assistência em amamentação, foi constatado o descolamento entre o modelo adotado pela instituição e a vivência das mulheres, traduzidas pela impossibilidade de cumpri-lo, frente às dificuldades impostas no seu cotidiano. Por fim, sugere a reformulação do modelo assistencial, de modo que leve em conta, além dos determinantes biológicos, os condicionantes sócio-culturais associados a essa prática. A dissertação de Veloso 13 retoma essa linha de investigação. Seu pressuposto foi de que o modelo de Alojamento Conjunto Especial (ACE) contribui para redução do desmame precoce entre a clientela assistida na UTI Neonatal da Maternidade Dona Evangelina Rosa. Seus resultados indicam que a adoção de práticas que visam a redução do tempo de permanência do prematuro na incubadora e a presença da mãe na instituição foram relevantes na melhoria dos índices de aleitamento materno. Crivaro, 14 preocupado com a assistência em 
amamentação no ambiente hospitalar, demonstrou que a prevalência de Aleitamento Materno (AM) foi inversamente influenciada pelo tempo de internação. Quanto maior o tempo de internação no ambiente da UTI-neonatal, menor a prevalência de AM. A pesquisa teve como enfoque o momento da alta dos recém-nascidos internados em unidades de tratamento intensivo neonatal, de Hospitais Amigos da Criança, no município do Rio de Janeiro. Em outra pesquisa qualitativa voltada para o assistir em amamentação, Castro, 15 com o referencial das representações sociais, estudou as questões de amamentação com profissionais de saúde que assistem gestantes e puérperas portadoras do vírus da imunodeficiência humana (HIV). Seu objetivo foi discutir a ambigüidade entre o incentivo à amamentação e a necessidade de inibir a lactação em tempos de epidemia de AIDS, através da revisão dos modelos assistenciais prescritos pelas políticas públicas de saúde. Cruz 16 trabalhou com o inédito tema relacionado ao posicionamento do pai frente à fase de gestação, parto e pós-parto, sobretudo no tocante à amamentação do filho. Seus resultados mostraram que os pais se posicionaram de uma maneira favorável diante da amamentação. Aponta que o pai pode ter um papel importante de apoio na fase de amamentação e recomenda que o mesmo deve ser incluído no programa de saúde da família, desde as primeiras consultas no pré-natal, para que os sentimentos de insegurança e ciúme não interfiram negativamente na amamentação do filho.

Vários e importantes trabalhos foram desenvolvidos com relação à formação e qualificação de profissionais para lidar com a questão da amamentação. O ensino do aleitamento materno na graduação de medicina foi investigado por Silva. $17 \mathrm{O}$ referencial teórico metodológico teve como base a proposta elaborada pela Organização Pan-Americana da Saúde (OPAS) e pela Organização Mundial da Saúde (OMS) para avaliação do ensino de aleitamento materno nas escolas de saúde do país no ano de 1993. O autor conclui que deve haver uma adequação do ensino com a assistência que integraliza a saúde da mulher, objetivando a capacitação do profissional médico para lidar com a questão do aleitamento materno de forma segura e eficaz. Também nesta linha de pesquisa encontra-se a dissertação de Monteiro, 18 que teve como objeto a representação que docentes e graduandos dos cursos de Nutrição têm sobre a amamentação. Na interpretação das falas dos entrevistados, o autor constatou que a mulher, no período de gravidez e amamentação foi identificada apenas como "mãe", sendo desqualificada, principalmente na fala dos alunos, por qual- quer comportamento que não correspondesse a um ideal de maternidade. Verificou ainda que na formação do nutricionista, no que diz respeito a amamentação, falta uma abordagem que contemple os aspectos sociais, induzindo a uma compreensão descontextualizada do processo. Por fim, é apontada a necessidade de refletir e avaliar a forma como é abordado o tema amamentação na graduação em Nutrição. A dissertação de Zein ${ }^{19}$ focaliza seu objeto na qualificação de recursos humanos. Seu campo de investigação foi o Curso de Aconselhamento em Amamentação, desenvolvido no Brasil pelo Ministério da Saúde, tendo por base a teoria das representações sociais. Sua conclusão é de que o curso, apesar de não apresentar transformações concretas, cria no profissional a oportunidade reflexiva de uma nova forma de pensar sobre sua postura no assistir a mulher que amamenta. Esse mesmo Curso foi tema da dissertação de Azevedo,20 que buscou identificar e analisar, a partir da compreensão de sua concepção teórico- pedagógica, os obstáculos que impedem a ação transformadora esperada por seus idealizadores. Em sua conclusão fica o entendimento de que o enfoque adotado no Curso, com relação a amamentação, termina por reduzir a ação do profissional como um ato de assistir, em função da excessiva valorização das questões técnicas. Além disso, conclui, a concepção pedagógica demonstra ser incompatível com a ação transformadora pretendida no modelo proposto.

O controle de qualidade em BLH se configura como outro tema contemplado na produção acadêmica recente. $O$ próprio crescimento da REDEBLH induziu a uma necessidade de maior ancoragem científica. Neste sentido, vale destacar o trabalho de Moreira, 21 realizado com base em informações oriundas de inspeções realizadas pela Vigilância Sanitária em onze BLH do Estado do Rio de Janeiro. Dentre outras questões, o autor conclui sobre a importância de adoção de práticas preventivas, com base na análise de risco, no intuito de assegurar a qualidade do leite humano distribuído. Ainda na mesma linha de controle de qualidade, Fonseca $^{22}$ identificou um vazio na literatura sobre Análise de Perigos e Pontos Críticos de Controle (APPCC) em BLH. Seus resultados revelam que o LHO pasteurizado - LHOp, em BLH da rede municipal de saúde do Rio de Janeiro, distribuído aos lactentes nas maternidades que fizeram parte do estudo, são processados em condições sanitárias adequadas. Silva 23 descreve as normas técnicas para coleta, processamento e distribuição de leite humano ordenhado, na perspectiva da Gestão pela Qualidade em BLH. Essa tese foi decorrente de demanda 
gerada com a expansão da REDEBLH no Brasil. Para o autor há necessidade de uniformização de condutas, visando o estabelecimento de um programa de garantia de qualidade de produtos e processos sob a responsabilidade dos BLH.

\section{Conclusões}

Apesar da REDEBLH ser gerada com essa denominação e desenho a partir de 1998, o movimento de construção desse modelo é anterior e historicamente determinado.

O conhecimento aparece como elemento que confere conectividade. Ou seja, a análise realizada, da gênese e evolução dos BLH, permite identificar que além dos movimentos dos atores sociais envolvidos, há uma dinâmica da produção do conhecimento que deve ser entendida.

Neste sentido, o estudo evidencia a importância real e potencial do conhecimento que vem sendo gerado nas suas diversidades de métodos e de objetos de investigação. A apropriação desse conhecimento, como elemento transformador de práticas, será determinada em sua intensidade pela capacidade de compartilhá-lo. Assim, torna-se relevante a identificação de caminhos que ampliem tal compartilhamento.

Quando se apresentam resultados, com validação acadêmica, de pesquisas que indicam, por exemplo, necessidades de modificações em programas oficiais, abre-se espaço concreto para uma discussão que vai além do limite dos modelos de serviços e se instala na instância do aparelho de Estado, responsável pela formulação da política pública para o setor.

\section{Referências}

1. Almeida JAG, Maia PRS, Novak FR. Os bancos de leite humano como suporte para a redução da mortalidade infantil: a experiência brasileira. In: Anais do II Congresso Uruguayo de Lactancia Materna; 2004 setembro 1-4; Montevideo, Uruguay. Montevideo: Sociedad Uruguaya de Pediatria; 2004. Disponível em: http://www.bvsam.cict. fiocruz.br/evcientif/2culm/2culm.htm [2005 fev 4]

2. Almeida JAG. A evolução dos bancos de leite no Brasil [videocassete]. Rio de Janeiro: Núcleo de Vídeo do Centro de Informação Científica e Tecnológica da Fundação Oswaldo Cruz; 1992.

3. Minayo MCS. Caminhos do pensamento: epistemologia e método. Rio de Janeiro: FIOCRUZ; 2002.

4. Almeida JAG. Amamentação: um híbrido natureza-cultura. Rio de Janeiro: FIOCRUZ; 1999.
O mesmo caminho pode ser vislumbrado para os estudos que abordam formação e qualificação de profissionais. A identificação do descolamento entre as instâncias formadoras e as prestadoras de serviços, via de regra devido a distorções em estruturas curriculares, tal qual diagnosticado em alguns estudos, sugere de modo idêntico, discussões que transcendem o lócus formador e explicitam novamente o necessário compartilhamento.

Outro aspecto que merece relevo, diz respeito ao ineditismo teórico/metodológico identificado em alguns estudos, que serviram de base para novas problematizações e formulações. Nesse sentido, os avanços alcançados pela REDEBLH foram fundamentalmente dependentes do compartilhamento do conhecimento, a partir do qual se tornou possível a geração de novos saberes e práticas.

A análise da gênese e evolução da REDEBLH permitiu identificar a importância de sua sede - o Centro de Referência Nacional - como instância catalizadora, que capta as necessidades de mudança e as processa, utilizando-as não só como elemento indutor do avanço científico e tecnológico, mas também para a retroalimentação da formulação de política pública em seu âmbito de atuação. Estas questões são processadas, gerando respostas cientificamente embasadas, que retornam ao demandante por intermédio de processos de conversão do conhecimento.

Por fim, vale destacar que o estudo permitiu identificar a potencial contribuição da REDEBLH para promoção de transformações sociais e na formulação da política pública voltada para área da saúde da mulher e da criança.

5. Almeida JAG, Novak FR. Banco de Leite Humano: fundamentos e técnicas. In: Anais do VIII Congresso Brasileiro de Nutrição e Metabolismo Infantil; 1994 julho; Porto Alegre, Brasil. Porto Alegre: Sociedade Brasileira de Pediatria;1994.

6. INAM (Instituto Nacional de Alimentação e Nutrição). Programa Nacional de Incentivo ao Aleitamento Materno: relatório. Brasília (DF): Ministério da Saúde; 1987.

7. Maia PRS, Novak FR, Almeida JAG, Silva DA. Bases conceituais para uma estratégia de gestão: o caso da rede nacional de bancos de leite humano. Cad Saúde Pública. 2004; 20: 1700-8.

8. Almeida JAG. Amamentação: repensando o paradigma [tese doutorado]. Rio de Janeiro: Fundação Oswaldo Cruz; 1998. 
9. Souza LMBM. Alimentação do lactente brasileiro - saber construído ou saber induzido: reflexões sobre os saberes e práticas da alimentação do lactente na pediatria brasileira [tese doutorado]. Rio de Janeiro: Fundação Oswaldo Cruz; 2003.

10. Ferreira SLC. A decisão materna e a alimentação do filho no pós-alta hospitalar [dissertação mestrado]. Rio de Janeiro: Fundação Oswaldo Cruz; 2003.

11. Souza LMBM. Promoção, proteção e apoio. Representações sociais em aleitamento materno [dissertação mestrado]. Rio de Janeiro: Fundação Oswaldo Cruz; 1996.

12. Ramos CV. Amamentação do discurso à prática: um estudo sobre a percepção de mulheres assistidas na maternidade Dona Evangelina Rosa - Teresina, Piauí [dissertação mestrado]. Rio de Janeiro: Fundação Oswaldo Cruz; 2000.

13. Veloso LFG. Alojamento conjunto especial: uma proposta alternativa [dissertação mestrado]. Rio de Janeiro: Fundação Oswaldo Cruz; 2003.

14. Crivaro ET. Estudo da prevalência de aleitamento materno em unidades de tratamento intensivo neonatal de hospitais amigos da criança no município do Rio de Janeiro [dissertação mestrado]. Rio de Janeiro: Fundação Oswaldo Cruz; 2002.

15. Castro RC. As contradições do assistir em amamentação Incentivo ao aleitamento materno versus inibição da lactação [dissertação mestrado]. Rio de Janeiro: Fundação Oswaldo Cruz; 2002.
16. Cruz MHS. Como o homem representa o processo de amamentação do seu filho [dissertação mestrado]. Rio de Janeiro: Fundação Oswaldo Cruz; 2002.

17. Silva VG. Programa de controle interno e externo de qualidade em bancos de leite humano [tese doutorado]. Rio de Janeiro: Fundação Oswaldo Cruz; 2004.

18. Monteiro KA. O nutricionista e a amamentação: formação e docência para uma prática profissional [dissertação mestrado]. Rio de Janeiro: Fundação Oswaldo Cruz; 2000.

19. Zein ET. Princípios do aconselhamento aplicados ao assistir em amamentação na perspectiva dos profissionais de saúde [dissertação mestrado]. Rio de Janeiro: Fundação Oswaldo Cruz; 2001.

20. Azevedo IGS. Aconselhamento aplicado ao assistir em amamentação: uma análise da proposta oficial [dissertação mestrado]. Rio de Janeiro: Fundação Oswaldo Cruz; 2004.

21. Moreira AS. Condições higiênico-sanitárias dos bancos de leite humano do Estado do Rio de Janeiro sob a ótica da vigilância sanitária [dissertação mestrado]. Rio de Janeiro: Fundação Oswaldo Cruz; 2000.

22. Fonseca LM. Estudo da implantação da metodologia de análise de perigos e pontos críticos de controle em bancos de leite humano no município do Rio de Janeiro [dissertação mestrado] Rio de Janeiro: Fundação Oswaldo Cruz; 2003.

23. Silva VG. O ensino do aleitamento materno na Universidade Federal de Juiz de Fora [dissertação mestrado]. Rio de Janeiro: Fundação Oswaldo Cruz; 1998.

Recebido em 7 de junho de 2005

Versão final apresentada em 8 de junho de 2006

Aprovado em 27 de junho de 2006 